\title{
Shades of spring
}

As winter thaws in the Northern hemisphere, a fresh supply of products speeds up $P C R$ analysis, generates more sequence more quickly, and balances centrifugal spins.

The Mouse $\mathrm{Kit}^{\mathrm{TM}}$, distributed by STRATAGENE, provides a toolbox for gene targeting in embryonic stem (ES) cells. The kit contains reagents optimized and manufactured by Lexicon Genetics Incorporated, a commercial producer of knockout mice. In addition to AB2.2-Prime ES Cells, the kit includes ESQ feeder cells and fetal bovine serum pre-qualified to optimally support the growth of the ES cells. The feeder cells have been engineered to be neomycin- and puromycin-resistant to maintain the ES cells during selection. Based on the restriction map profile of the region of interest, the user can select the appropriate vector from STRATAGENE's pKO Scrambler Series ${ }^{\circledR}$ of gene targeting vectors for subcloning the region targeted for homologous recombination. The series, comprised of 36 vectors, contains both positive (neo and puro) and negative (thymidine kinase or diptheria toxin) selection cassettes. Negative markers are incorporated only when non-homologous recombination occurs, which reduces the selection of false positives tenfold.

The Mouse on Mouse (M.O.M. ${ }^{\mathrm{TM}}$ ) Immunodetection Kit from Vector Laboratories, Inc. provides a means of minimizing background staining. Immunohistochemical analysis in the mouse is frequently impeded by the inability of anti-mouse sec-

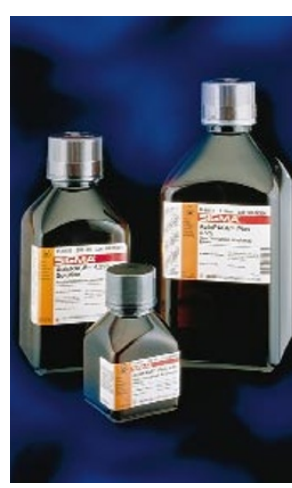

SIGMA's high-throughput acrylamide gel solutions ase VII to recognize DNA mismatches and cleave at the site where a 'bubble' is formed by mispairing, insertion or deletion of bases. The reference and test samples of DNA are amplified, hybridized and incubated with T4 endonuclease VII, followed by separation by gel electrophoresis. The PASSPORT ${ }^{\mathrm{TM}}$ kit can analyse fragments of several kilobases, thereby offering an advantage over traditional methods of genetic mutation analysis, such as direct sequencing or single-strand conformational polymorphism (SSCP) analysis. According to the manufacturer, the kit has a high sensitivity for detecting heterozygous mutations, with mutation signals detectable at $10 \%$ and lower in mixed backgrounds.

SIGMA's high-throughput acrylamide gel solutions, developed in collaboration with Washington University Genome Sequencing Center, is claimed to provide more sequence in less time. Gel runs with AutoPAGE $^{\mathrm{TM}} \quad$ Plus $\quad 4.5 \%$ reveal 100 bases per hour, and enable read lengths of 700-800 bases in approximately 7 hours. AutoPAGE ${ }^{\mathrm{TM}}$ $4.25 \%$, formulated for shorter read lengths, provides $450-500$ bases in as little as 2.5 hours.

According to its manufacturer, the LightCycler ${ }^{\mathrm{TM}}$ from Roche Diagnostics Ltd is the world's fastest thermal cycler, ondary antibodies to distinguish between the primary mouse antibody and endogenous mouse immunoglobulins. M.O.M. utilizes a proprietary agent that blocks endogenous murine immunoglobulins, thereby reducing undesired background staining. The blocking agent is used in conjunction with a biotinylated anti-mouse IgG secondary antibody and Vector Labs' peroxidasebased VECTASTAIN ${ }^{\circledR}$ Elite ABC kit.

The PASSPORT ${ }^{\mathrm{TM}}$ kit from Amersham Pharmacia Biotech can be used to detect mutations and polymorphisms. The kit, an enzymatic mutation detection (EMD) assay, exploits the ability of T4 endonucleproducts can be monitored using SYBR ${ }^{\circledR}$ Green I Dye, which preferentially binds double-stranded DNA and emits a signal

when bound that is proportional to the dsDNA concentration. An alternative quantification method, based on fluorescence resonance energy transfer (FRET), uses two hybridization probes, one with a 5 ' donor fluor and the

other with a 3 acceptor fluor. These probes are internal to the amplification primers and to hybridize in close proximity to each other.

The donor Roches lightcycler fluorophore of light to faster PCR analysis. is excited by

an external light source and transfers energy to the acceptor fluorophore, which in turn emits a light signal. In March, Roche plans to release an additional dye and software to support multiplex PCR analysis.

Oxford Molecular's MacVector ${ }^{\mathrm{TM}}$ 6.5, the latest sequence analysis software, offers user-defined primer testing, which evaluates the suitability of primer sequences for PCR or sequencing as well as predicting secondary structure formation and denaturation temperatures. The software also includes an expanded toolbox for protein sequence analysis, including algorithms for hydrophobicity and antigenicity. The user can save results as PICT files that can then be imported into graphics programs such as Adobe Photoshop, Illustrator and Microsoft PowerPoint, and the Clustal $\mathrm{W}$ multiple sequence alignments can now be printed in colour.

Providing a sense of balance are the first self-balancing rotors from Beckman Coulter, Inc. Designed for the J6 Series high-speed centrifuges, the JS4.2A and JS-4.2SMA ARIES rotors automatically detect and correct imbalances, eliminating the need for manual sample balancing. The Smart Balance technology can correct an imbalance even when a tube breaks during a run, allowing completion of the centrifuge program.

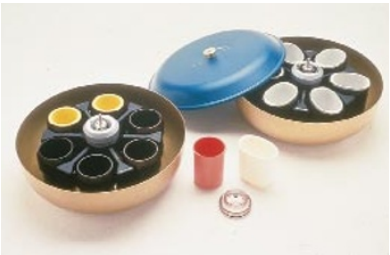

Maintaining balance with ARIES rotors from Beckman Coulter, Inc. 